\title{
Fluorescence Imaging Study of Transition in Underexpanded Free Jets
}

\author{
Jennifer A. Wilkes* ${ }^{\dagger}$, Paul M. Danehy ${ }^{\dagger}$, and Robert J. Nowak ${ }^{\dagger}$ \\ *Department of Physics, The College of William and Mary, Williamsburg VA 23187 USA, \\ email address: jennifer.a.wilkes@nasa.gov \\ ${ }^{\dagger}$ NASA Langley Research Center, Hampton VA, 23681-2199
}

Keywords: laser-induced fluorescence, turbulence, transition

\begin{abstract}
Planar laser-induced fluorescence (PLIF) is demonstrated to be a valuable tool for studying the onset of transition to turbulence. For this study, we have used PLIF of nitric oxide (NO) to image underexpanded axisymmetric free jets issuing into a low-pressure chamber through a smooth converging nozzle with a sonic orifice. Flows were studied over a range of Reynolds numbers and nozzle-exit-to-ambient pressure ratios with the aim of empirically determining criteria governing the onset of turbulence. We have developed an image processing technique, involving calculation of the standard deviation of the intensity in PLIF images, in order to aid in the identification of turbulence. We have used the resulting images to identify laminar, transitional and turbulent flow regimes. Jet scaling parameters were used to define a rescaled Reynolds number that incorporates the influence of a varying pressure ratio. An empirical correlation was found between transition length and this rescaled Reynolds number for highly underexpanded jets.
\end{abstract}

\section{INTRODUCTION}

Highly underexpanded axisymmetric free jets are important flows of interest for fundamental fluid mechanical studies as well as for practical applications. Of particular importance is an understanding of the development of instabilities in these flows. While the literature contains extensive studies of the transition to turbulence in boundary layers of flat plate flows, the literature containing experimental studies of transition in highly underexpanded axisymmetric free jets is considerably more sparse [1-6]. Understanding the stability characteristics of such flows became the motivation for this work, conducted as part of the NASA Shuttle Return to Flight (RTF) investigation into the effects of flow through vehicle wing breaches.

While CFD (computational fluid dynamics) codes can simulate turbulent flow, accurately predicting the transition from laminar to turbulent flow remains an unsolved problem. The empirical correlation resulting from these tests can therefore potentially be used to determine where turbulence models should be implemented in CFD simulations as well as providing data for comparison with CFD results.

Flow visualization was required for these tests. The flow environments encountered in these tests include regions of low static pressure, turbulent and/or three-dimensional flow structures, and regions of interest that lack strong density gradients. Such conditions, though frequently encountered in aerospace simulation facilities, cannot be satisfactorily visualized using traditional path-averaged techniques such as schlieren and shadowgraph, which rely on sufficiently strong density gradients. An alternative approach is therefore required to visualize these types of flows. Planar laser-induced fluorescence (PLIF) is a powerful flow visualization technique that provides a means of making non-intrusive measurements with sub-millimeter spatial resolution and flow-stopping temporal resolution in many of these challenging testing regimes. [7]

\section{PLIF MEASUREMENT System}

\section{A. Test Apparatus}

Tests were conducted at NASA Langley Research Center using the test section of the 15-Inch Mach 6 Wind Tunnel as a vacuum chamber. Gas bottles were stored in a ventilated, alarmed toxic gas cabinet. Nitrogen $\left(\mathrm{N}_{2}\right)$ seeded with $0.5 \%$ nitric oxide (NO) was plumbed into a heated $(\sim 500 \mathrm{~K})$ stainless steel plenum, through a converging nozzle with a nominal exit Mach number of 1, into the vacuum chamber. Mass flow controllers adjusted the flow rates. A schematic including these elements of the test apparatus is shown in Fig. 1.

Two parameters were varied: Reynolds number $\left(R e_{\text {exit }}\right)$ and jet pressure ratio $(J P R)$. Re exit was defined in terms of the nozzle exit diameter, $D_{e}$, as well as the density $\rho_{e}$, velocity $V_{e}$, and dynamic viscosity $\mu_{e}$ at the nozzle exit, as given by (1):

$$
\operatorname{Re}_{e x i t}=\frac{\rho_{e} V_{e} D_{e}}{\mu_{e}} .
$$

(Note that in keeping with conventions found in the literature, data are plotted in terms of a unit Reynolds number, with units of $\mathrm{m}^{-1}$. The unit exit Reynolds number is simply $R e_{e x i t} / D_{e}$. The nozzle used in these tests had an exit diameter of $D_{e}=2.4$ $\mathrm{mm}$.) $R e_{\text {exit }}$ was varied by changing the mass flow rates.

$J P R$ was defined as the ratio of the static pressure at the nozzle exit, $p_{e}$, to the ambient pressure in the vacuum chamber, $p_{a}$, according to (2):

$$
J P R=\frac{p_{e}}{p_{a}} .
$$






Figure 1. Schematic of experimental setup. Gas is plumbed through a heated plenum and nozzle into a vacuum chamber. A laser sheet enters the top of the vacuum chamber and excites nitric oxide molecules in the flow. An intensified CCD camera positioned at right angles to the laser sheet images the fluorescence.

$J P R$ was varied by changing the pressure in the vacuum chamber $\left(p_{a}\right)$. For a given $R e_{\text {exit }}$, the maximum attainable JPR was limited by the minimum attainable pressure in the vacuum chamber, which was about $130 \mathrm{~Pa}$ (1 torr).

A thermocouple and a pressure transducer measured the gas temperature $T_{0}$ and pressure $p_{0}$ in the plenum, upstream of the converging nozzle. Measuring these quantities made it possible to calculate conditions at the nozzle exit, and therefore the exit Reynolds number. Nozzle exit temperature $T_{e}(\mathrm{~K})$ and velocity $V_{e}(\mathrm{~m} / \mathrm{s})$ can be calculated from these measured quantities by assuming inviscid adiabatic flow and an exit Mach number of one, according to (3) and (4) [8]:

$$
\begin{gathered}
T_{e}=\frac{T_{0}}{1+\left(\frac{\gamma-1}{2}\right)} \\
V_{e}=\sqrt{\gamma R T_{e}}
\end{gathered}
$$

where $\gamma$ is the ratio of specific heats of the gas $(\gamma=1.4$ for nitrogen), and $R$ is the specific gas constant with a value of $287 \mathrm{~J} /(\mathrm{kg} \cdot \mathrm{K})$. Furthermore, with the added assumption of isentropic flow, nozzle exit pressure $p_{e}(\mathrm{~Pa})$ and density $\rho_{e}$ $\left(\mathrm{kg} / \mathrm{m}^{3}\right)$ can be calculated using (5) and (6) [8]:

$$
p_{e}=\frac{p_{0}}{\left(1+\frac{\gamma-1}{2}\right)^{\gamma /(\gamma-1)}}
$$

$$
\rho_{e}=\frac{p_{0}}{R T_{0}\left(1+\frac{\gamma-1}{2}\right)^{1 /(\gamma-1)}} .
$$

The dynamic viscosity $\mu_{e}(\mathrm{~Pa} \cdot \mathrm{s})$ at the nozzle exit was calculated using the power law approximation given in (7):

$$
\mu_{e}=\mu_{r e f}\left(\frac{T_{e}}{T_{r e f}}\right)^{n}
$$

This power law accounts for the effect of temperature on dynamic viscosity while neglecting the slight dependence on pressure. Reference [9] gives an empirical value of $n=0.67$ for nitrogen, where $T_{r e f}=273 \mathrm{~K}$ and $\mu_{r e f}=1.663 \times 10^{-5} \mathrm{~Pa} \cdot \mathrm{s}$. Values for nitrogen were used because the gas consisted of $99.5 \% \mathrm{~N}_{2}$, and because the $\mathrm{NO}$ that made up the remaining $0.5 \%$ has a viscosity similar to that of $\mathrm{N}_{2}$ [9].

\section{B. Laser System}

The laser system consists of three main components: a pump laser, a pulsed dye laser (PDL), and a wavelength extender (WEX). An injection-seeded Nd:YAG laser operating at $10 \mathrm{~Hz}$ pumps a tunable PDL. The output of the dye laser and the residual infrared from the $\mathrm{Nd}$ :YAG are combined in a WEX containing both a doubling and a mixing crystal. The resulting output is tuned to a wavelength of $226.256 \mathrm{~nm}$, chosen to excite the strongly fluorescing spectral lines of NO near the $\mathrm{Q}_{1}$ branch head. In this notation, $\mathrm{Q}$ indicates that the rotational quantum number $J$ is the same for both the upper and lower states of the transition; i.e., $\Delta J=0$. The subscript 1 indicates that, in both the upper and lower states involved in the transition, $J$ is related to $N$ by $J=N+1 / 2$, where $N$ is the quantum number for total angular momentum, excluding spin.

A monitoring gas cell system is used to ensure that the laser is tuned to the correct spectral line of NO. The gas cell contains a low-pressure mixture of $5 \% \mathrm{NO}$ in $\mathrm{N}_{2}$. A quartz window serves as a beam splitter and sends a small portion of the laser energy through windows on either side of the gas cell. A photomultiplier tube (PMT) monitors the fluorescence intensity through a third window at right angles to the path of the laser beam.

The components of this laser system are mounted within a two-level, enclosable, portable cart. A photograph of this portable PLIF system is shown in Fig. 2 with the panels removed to show the internal components. When all of the panels are in place, a single monochromatic ultraviolet laser beam exits the cart, creating a relatively safe operating environment.

Optics form the beam into a $100 \mathrm{~mm}$ wide $\mathrm{x} \sim 1 \mathrm{~mm}$ thick laser sheet, which is directed vertically downward through a window in the top of the test section. Fluorescence is imaged onto a gated, intensified CCD at a viewing angle normal to the laser sheet. A $1 \mathrm{~mm}$ thick [Schott glass UG5] filter was 


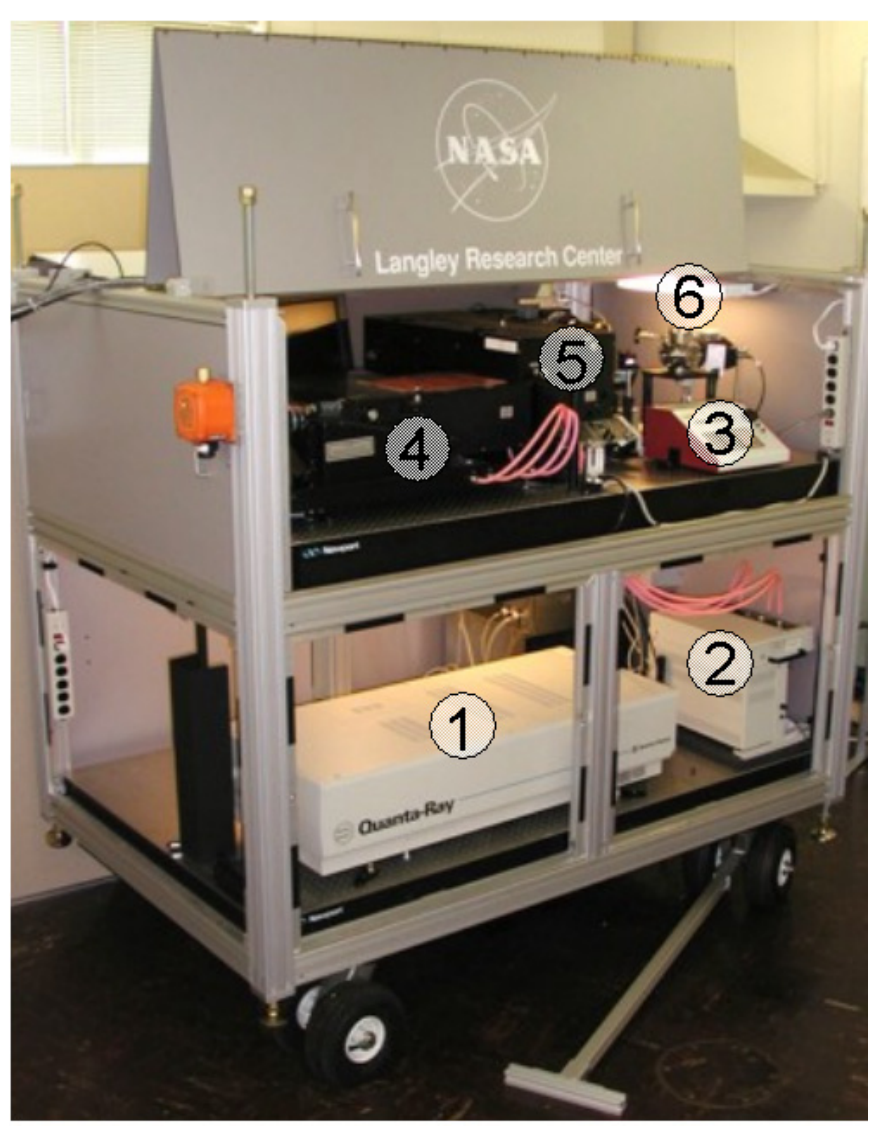

Figure 2. The portable PLIF system, shown with panels removed. Components include: (1) Nd:YAG laser; (2) dye circulators with Rhodamine 590 and Rhodamine 610 laser dyes in a methanol solvent; (3) wavelength controller for the (4) pulsed dye laser; (5) wavelength extender; and (6) lowpressure monitoring gas cell.

placed in front of the camera lens in order to attenuate scattered light at the laser's frequency. The schematic of the experimental setup shown in Fig. 1 includes these elements as well.

\section{Image Acquisition}

Images were acquired at $10 \mathrm{~Hz}$ with a $1 \mu$ s camera gate and a spatial resolution of 7 pixels $/ \mathrm{mm}$. This temporal and spatial resolution was more than sufficient to resolve turbulent flow structures of interest. Further details of the laser and camera system can be found in [10].

Sets of 100 single-shot images were acquired for a range of unit $\operatorname{Re}_{\text {exit }}\left(0.07 \times 10^{6} \mathrm{~m}^{-1}\right.$ to $\left.14.8 \times 10^{6} \mathrm{~m}^{-1}\right)$ and $J P R$ (1.8 to 38). Background images were also acquired on each day of testing for a range of vacuum chamber pressures. During the acquisition of these background images, the laser was firing but no gas was flowing through the nozzle. Any nonzero intensity in these background images is due to either camera dark current or the laser scatter and room light not blocked by the filter in front of the camera lens. Background images were created from the average of 100 single-shot images in order to smooth out random shot-to-shot variations in background intensity.

\section{PLIF Intensity}

The absolute intensity at any given pixel in a PLIF image has a functional dependence on many local flow parameters as given by (8):

$$
S_{f} \propto E \rho \Phi \chi_{N O}\left[\sum f_{B} B_{12} g_{i}\right]
$$

where $S_{f}$ is the fluorescence signal; $E$ is the energy in a single laser pulse; $\rho$ is the local gas density; $\Phi$ is the fluorescence yield; $\chi_{\mathrm{NO}}$ is the local mole fraction of NO; $f_{B}$ is the Boltzmann fraction; $B_{12}$ is the Einstein absorption coefficient for the probed transition; and $g_{i}$ is the spectral overlap integral. $\Phi, f_{B}$, and $g_{i}$ are functions of the local temperature; $\Phi$ and $g_{i}$ also depend on the local pressure. Since the fluorescence intensity depends on so many local flow parameters, variations in any one of these parameters will affect the resulting intensity in a PLIF image. While this means that the interpretation of PLIF images is not always straightforward, it also means that PLIF images are well suited to detecting fluctuations within a flowfield.

\section{E. Image Processing and Analysis}

Single-shot images were processed to correct for background scattered light and camera dark current as well as spatial variations in laser sheet intensity. Both the background image and the single-shot images were smoothed with a filter (a 3 pixels $x 3$ pixels rotationally symmetric low-pass Gaussian filter with a standard deviation of 1) prior to additional processing in order to reduce noise in the images. A background image was then subtracted from the filtered single-shot image. Images were subsequently corrected for laser nonuniformities. Conveniently, jet gas containing nitric oxide diffused relatively uniformly into the test chamber in regions away from the jet. The fluorescence from the diffuse nitric oxide in these regions provided a suitable laser-energy reference, allowing the spatial variation in the laser intensity to be corrected in the images. This was accomplished by selecting an area of the image above the core of the jet flow and finding the average intensity along each column in that region. (Note that raw images were 512 x 512 pixels; images presented in Figs. 3, 7, 8 and 9 have been cropped to show the regions of greatest interest.) Finally, the backgroundcorrected images were divided by this laser sheet profile. Since the laser sheet is typically brightest in the center of the sheet, the result of dividing an image by the laser sheet profile was to increase the brightness of the left and right edges of the image relative to the central part of the image.

After background subtraction and laser-sheet correction, each set of 100 images was processed to create an averaged image at that flow condition. Single-shot images were then compared to this averaged image. A map was created showing the standard deviation of the fluorescence intensity at each pixel in the images. This map was generated by first subtracting the averaged image from each single-shot image, squaring the difference at each pixel, averaging the squared 
differences of all 100 images, and finally, taking the square root of this average value at each pixel. The resulting map is then an indication of how much the intensity varied on average at a given location in the flow. Regions with small variations were interpreted to correspond with laminar flow, while the large variations in other regions were attributed to some degree of turbulence.

\section{EXPERIMENTAL RESULTS}

Fig. 3 shows a typical image sequence. The top image is a single-shot image. Towards the right of the image, turbulent structures are clearly visible. The middle image in the sequence shows the average of 100 single-shot images taken at this same flow condition. The bottom image is the map showing the standard deviation of the intensity of 100 singleshot images at each pixel.

For the purposes of this study, we chose to define laminar regions of the flow as those where fluctuations within the flow were comparable or less than the background fluctuations in regions outside the core flow of the jet. The onset of transition is then defined to occur at the downstream location where fluctuations begin to rise significantly above the background level. This is marked as $x_{\text {trans }}$ in Fig. 3. Finally, we identified the flow as being fully turbulent downstream of the location where instabilities have penetrated the core of the jet, as evidenced by an increase in the standard deviation at the jet centerline. This location is labeled $x_{\text {turb }}$ in Fig. 3. For jets that remained laminar within the field of view of the camera, the distance from the nozzle exit to the rightmost edge of the image was identified as $x_{\text {lam }}$.

For each test condition, the standard deviation image and

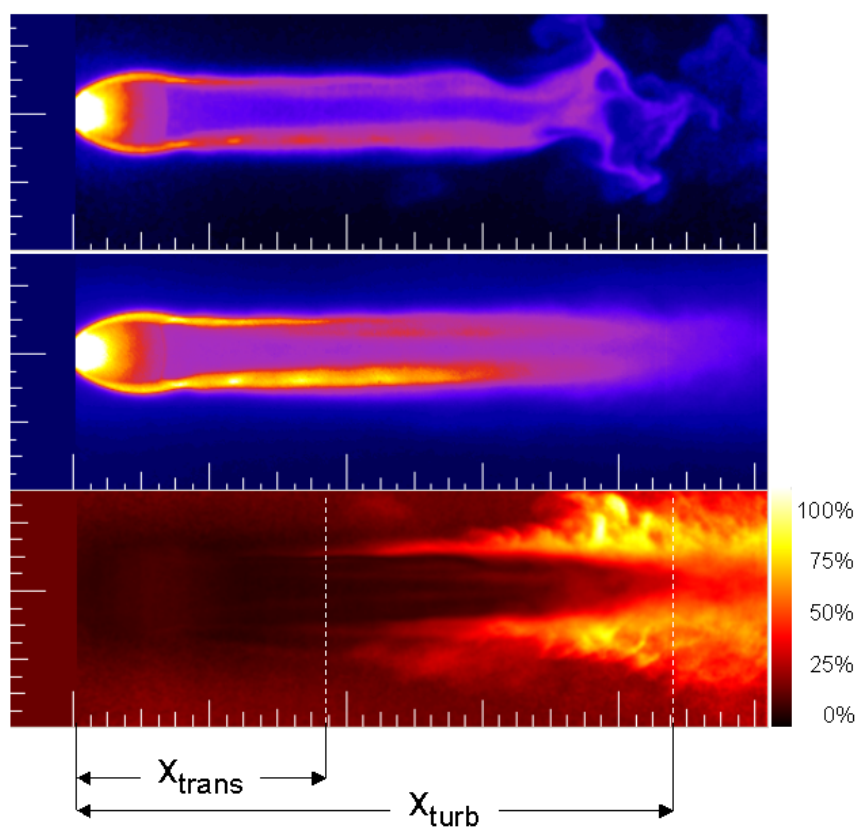

Figure 3. (top) Single-shot image of PLIF intensity; (middle) 100-shot average image for unit $R e_{\text {exit }}=2.7 \times 10^{6} \mathrm{~m}^{-1}$ and $J P R=16$; (bottom) standard deviation of PLIF intensity with transitional and turbulent locations marked. the single-shot images were examined. The flow was then labeled laminar, transitional, or turbulent based on the state of the flow at the most downstream location within the field of view of the camera. For laminar flows, $x_{\text {lam }}$ was recorded; for transitional flows, $x_{\text {trans }}$ was recorded. For turbulent flows, both $x_{\text {trans }}$ and $x_{\text {turb }}$ were recorded. The test matrix is plotted in Fig. 4, showing the range of Reynolds numbers and jet pressure ratios covered during the test.

Most data were acquired with a $64 \mathrm{~mm}$ field of view. For selected test conditions, a wider field of view $(165 \mathrm{~mm})$ was employed in order to determine the jet behavior further downstream. A flow condition that appeared transitional within the smaller field of view might have become fully turbulent within the wider field of view. Test points are shown for both the $64 \mathrm{~mm}$ and $165 \mathrm{~mm}$ fields of view; therefore some test points are labeled with two labels (e.g. the test point for $J P R=4.3$ and unit $R e_{e x i t}=0.6 \times 10^{6} \mathrm{~m}^{-1}$ is labeled as both transitional and turbulent).

In Fig. 4, points above a certain Reynolds number (about 3 $\mathrm{x} 10^{6} \mathrm{~m}^{-1}$ ) appear to become fully turbulent, while points below a certain Reynolds number (about $0.3 \times 10^{6} \mathrm{~m}^{-1}$ ) appear to remain laminar, which was the expected trend. However, this leaves a Reynolds number rang-spanning about an order of magnitude - over which flows exhibit mixed behavior. Clearly, additional parameters besides $J P R$ and $R e_{\text {exit }}$ need to be considered, not only to predict if a flow will become turbulent, but also at what downstream distance the transition to turbulence will occur.

These same data are plotted in Fig. 5 in terms of unit exit Reynolds number versus downstream distance, rather than jet pressure ratio. The general and expected trend appearsflows with lower unit Reynolds numbers are more likely to remain laminar. However, a clear relationship indicating the distance at which one might expect a given flow to undergo transition to turbulence is not readily apparent. For a many values of $R e_{\text {exit }}$, transition occurred at different distances for different test conditions. For instance, some flows with a unit Reynolds numbers near $1 \times 10^{6} \mathrm{~m}^{-1}$ became fully turbulent in less than $20 \mathrm{~mm}$, while others remained laminar beyond 160

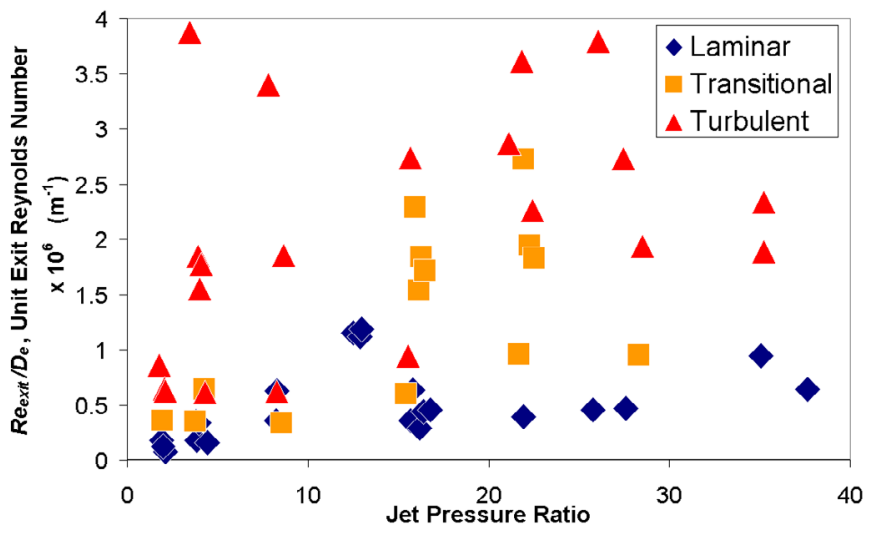

Figure 4. Unit exit Reynolds number versus jet pressure ratio. This plot shows the range of conditions over which these tests were conducted. A data point with a unit exit Reynolds number of nearly 15 and a $J P R$ of 7.5 is not shown. 


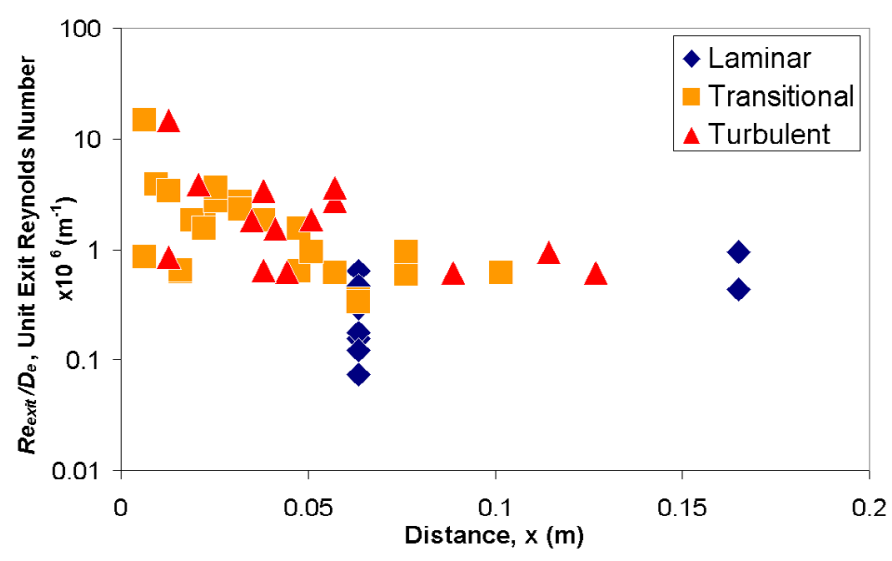

Figure 5. Unit exit Reynolds number versus distance downstream of the nozzle exit.

mm. Note that laminar data fall at one of two distances; these correspond to the extent of the camera's field of view, either $64 \mathrm{~mm}$ or $165 \mathrm{~mm}$.

The lack of an obvious correlation in Fig. 5 led us to postulate that the jet behavior was a function not only of Reynolds number, but of jet pressure ratio as well. Scaling laws described by Yüceil and Ötügen in Ref. [11] provide a means of redefining the Reynolds number to include the effects of JPR. Conditions at the nozzle exit serve as initial conditions for defining an "equivalent jet." They assume that the initial jet is allowed to expand adiabatically until the pressure in the jet reaches the ambient pressure. At this point - which they call location 2-all of the flow conditions are recalculated. Equation (7) can be combined with the equations for rescaled velocity $V_{2}$, density $\rho_{2}$, and temperature $T_{2}$ from Ref. [11] to arrive at an expression for the rescaled unit Reynolds number in terms of $J P R$ and nozzle exit conditions. This expression is given in Eq. (9):

$$
\begin{gathered}
\text { unit } \mathrm{Re}_{2}=\frac{\rho_{2} V_{2}}{\mu_{2}} \\
=\frac{\operatorname{Re}_{\text {exit }}}{D_{e}} \frac{2 \gamma\left(\gamma M_{e}^{2} J P R+J P R-1\right)\left(2 \gamma^{2} M_{e}^{2} J P R^{2}\right)^{n}}{\left.2 \gamma M_{e}^{2} J P R(\gamma+J P R-1)-(\gamma-1)(J P R-1)^{2}\right]^{n+1}} .
\end{gathered}
$$

Fig. 6 shows the data plotted in terms of this rescaled unit Reynolds number, unit $R e_{2}$. The relationship between this rescaled Reynolds number and transition length is more definitive. Independent power law fits to the transitional and turbulent data give $R^{2}$ values of better than 0.7 . It is also important to note that all laminar points now lie below the fitted curve for turbulent test points. This indicates that, for a given $R e_{2}$, there exists a distance beyond which the flow will likely be fully turbulent, and below which the flow will likely remain laminar.

While attempting to find any systematic errors in the data, we reexamined the physical structures of the flows under investigation. We observed that flows with jet pressure ratios of less than about 3 exhibited different physical characteristics

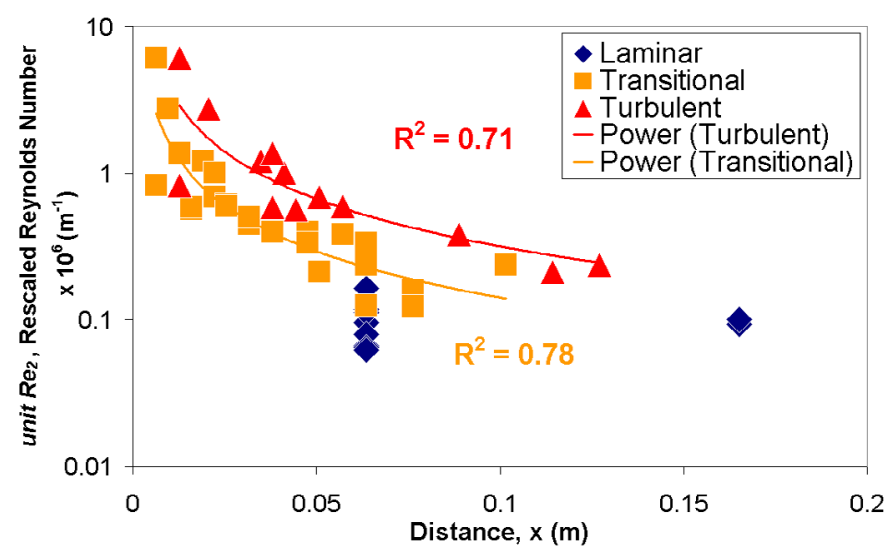

Figure 6. Correlation between rescaled unit Reynolds number and the distance downstream at which the jet flow became transitional (orange squares) and turbulent (red triangles). Power law fits to the data are shown.

This plot includes data for jet pressure ratios ranging from 1.8 to 38 .


Figure 7. Single-shot PLIF images of underexpanded jet flows at five different jet pressure ratios. From top to bottom, these $J P R$ s are: $36,22,8,5$, and 2. The presence of a Mach disk characterizes high JPR flows, while low $J P R$ flows are characterized by a diamond shock pattern. Mach disks were observed in flows with $J P R \mathrm{~s}$ greater than about 3. 
than flows with higher JPRs. Fig. 7 contains images of flows at five different jet pressure ratios. For the top four cases, flow structures including a barrel shock structure, a Mach disk and a shear layer can be seen. In the fourth image, the Mach disk is fairly small, and the flow is beginning to exhibit diamond shock features. In the bottom case, a Mach disk is no longer evident; the flow instead consists of a diamond shock pattern.
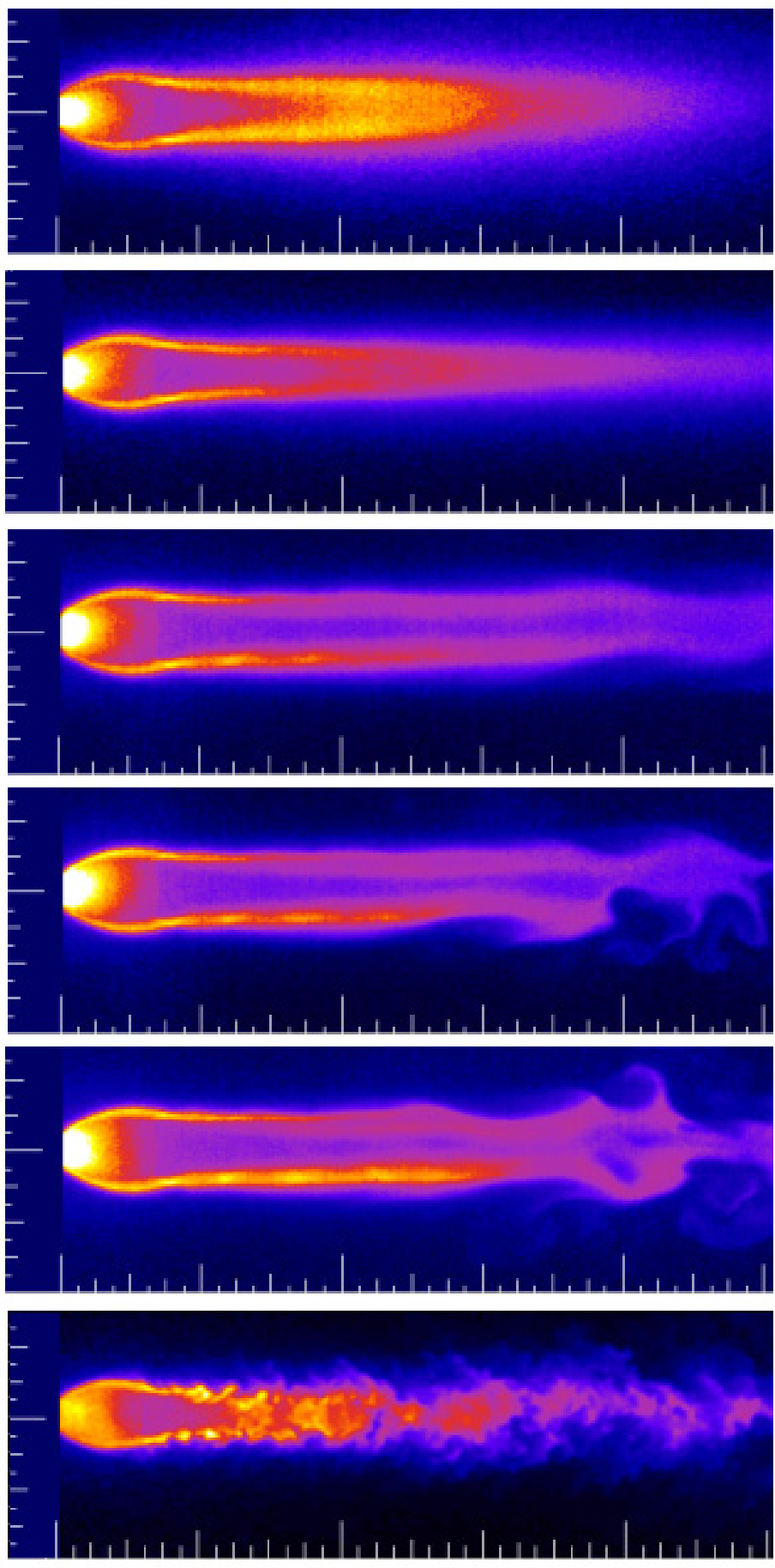

Figure 8. The effect of Reynolds number on transition to turbulence for a jet pressure ratio of about 16. Single-shot PLIF images (left) are displayed along with the corresponding standard deviation maps (right) for six values of unit $R e_{\text {exit }}$ (top to bottom): $0.62,0.95,1.8,2.3,2.7$, and $14.8 \times 10^{6} \mathrm{~m}^{-1}$.

When and if flows with high jet pressure ratios become turbulent, it appears that instabilities first develop in the shear layer, growing until they reach the core of the jet. One such case was illustrated in Fig. 3 for a flow with a JPR of 16. Fig. 8 illustrates the effect that Reynolds number has on the location of the onset of transition for flows with JPRs of about 16. The general trend is for flows at higher Reynolds numbers to undergo transition in a shorter distance.
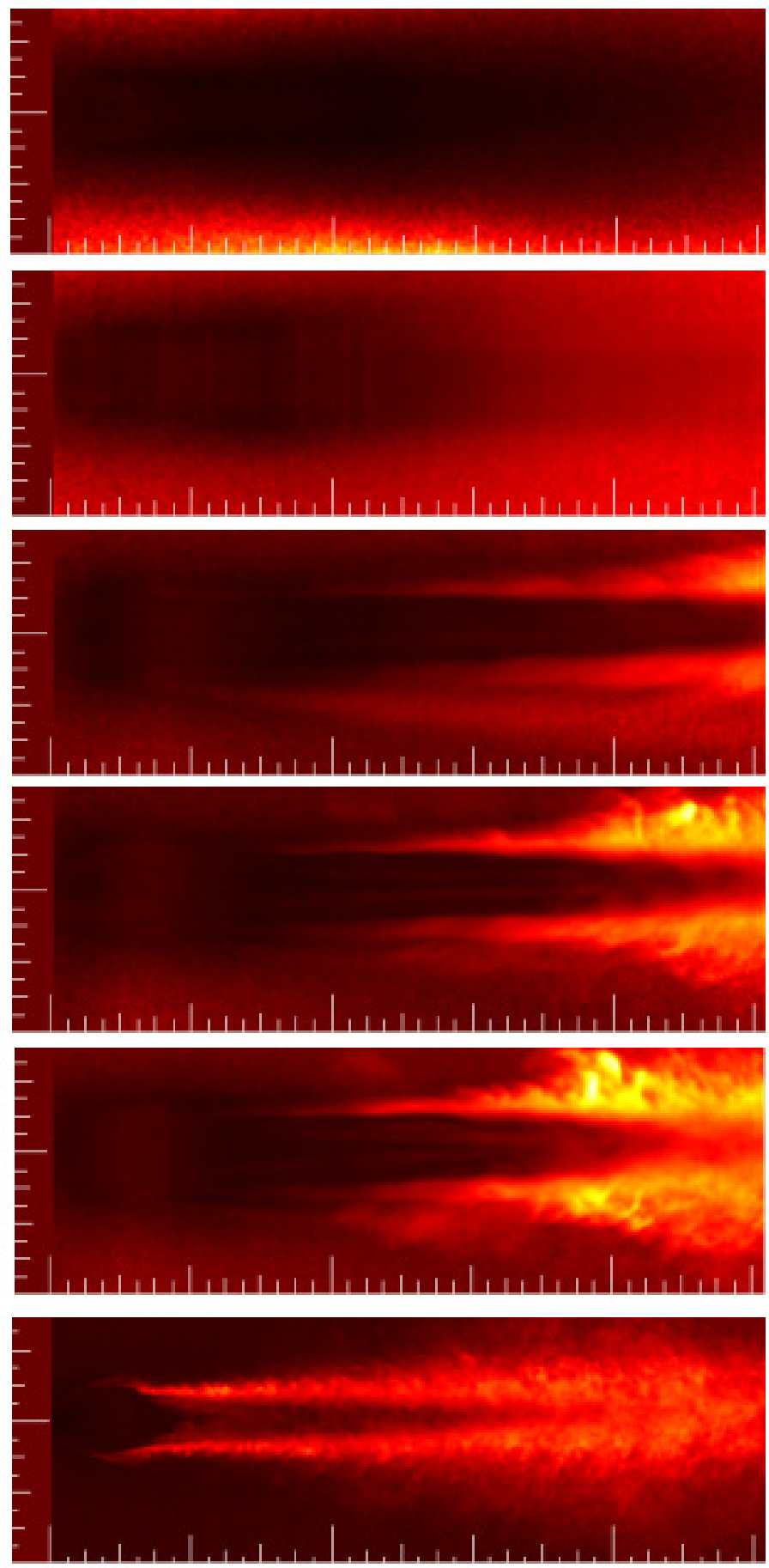




Figure 9. PLIF image illustrating the "pinching off" mode to turbulence, characteristic of flows distinguished by a diamond shock pattern. This flow has a $J P R=2$. See Fig. 3 for an illustration of a turbulent flow at a $J P R=16$.

Flows with low jet pressure ratios seemed to exhibit a different pattern when and if they became turbulent. Fig. 9 illustrates this for a flow with a $J P R$ of 2 . As the flow becomes unstable, the diamond shock pattern appears to become distorted, and globules of jet fluid begin to be pinched off.

Donaldson and Snedeker use a $J P R$ of approximately 2 to delineate the boundary between moderately underexpanded and highly underexpanded jets [1]. They also note that the differences in physical flow structures - such as the appearance of a slip line and shear layer in highly underexpanded jets - affect jet diffusion, spreading, mixing, and dissipation rates.

These physical differences between flows of different pressure ratios led us to consider only data points corresponding to JPRs of greater than 3 in our final correlation. The final correlation that was found between the rescaled unit Reynolds number and transition length is displayed in Fig. 10. The lower line shows the correlation between the rescaled Reynolds number and distance to transition; the upper line shows the distance to turbulence. Scatter in the data has been reduced by limiting the correlation to highly underexpanded jets with $J P R$ s greater than 3 .

From these correlations, one can predict whether a given underexpanded jet will remain laminar over a certain distance,



Figure 10. Correlation between rescaled unit Reynolds number and the distance downstream at which the jet flow became transitional and turbulent. Data for jet pressure ratios less than 3 are not included here. or whether turbulent effects will become manifest. These predictions can provide valuable information to researchers running CFD simulations of such flows, indicating whether turbulence models need to be included in the simulation of a given flow. The correlations also help to define the range over which underexpanded jet flows will be neither fully laminar nor fully turbulent, regions where experimental measurements may prove to be most critical.

\section{CONCLUSION}

In this work, we have demonstrated a powerful image processing technique to aid in the identification of turbulence using PLIF flow visualization images. This technique highlights turbulent regions of a flow by visualizing the standard deviation of fluorescence intensity throughout the flow. The image processing technique employed in this work could also readily be extended to studying transition and/or turbulence effects in other flows, particularly for tests in which spatially and temporally resolved flow visualization data is desired. In the current study, we have used the information gathered from such images to identify a correlation between a rescaled Reynolds number and downstream distance to transition for highly underexpanded axisymmetric sonic free jet flows.

\section{ACKNOWLEDGEMENTS}

The authors wish to acknowledge the collaborative input of Scott Halloran and Don Picetti of The Boeing Company and Chris Glass of NASA Langley Research Center, as well as the technical assistance of David Alderfer, Stephen Jones, and Paul Tucker of NASA Langley Research Center. This work was funded as part of the Shuttle Return to Flight effort through Chuck Campbell of NASA Johnson Space Center and Tom Horvath of NASA Langley Research Center.

\section{REFERENCES}

[1] C. D. Donaldson and R. S. Snedeker, "A study of free jet impingement. Part 1. Mean properties of free and impinging jets," J. Fluid Mech., vol. 45, part 2, pp. 281-319, 1971.

[2] C. D. Donaldson, R. S. Snedeker, and D. P. Margolis, "A study of free jet impingement. Part 2. Free jet turbulent structure and impingement heat transfer," J. Fluid Mech., vol. 45, part 3, pp. 477-512, 1971.

[3] E.S. Love, C.E. Grigsby, L.P. Lee, and M. J. Woodling, "Experimental and theoretical studies of axisymmetric free jets," NASA Technical Report, R-6, 1959.

[4] E. J. List, "Turbulent jets and plumes," Ann. Rev. Fluid Mech., vol. 14, pp. 189-212, 1982.

[5] D.C. Fourguette, M.G. Mungal, and R.W. Dibble, "Time evolution of the shear layer of a supersonic axisymmetric jet," AIAA Journal, vol. 29, no. 7, pp. 1123-1130, July 1991.

[6] R. E. DeOtte, Jr., G.L. Morrison, and R. D. Sewell, "LDV measurements of the velocity field in an underexpanded supersonic jet $(\mathrm{Ma}=1.5)$, " AIAA $30^{\text {th }}$ Aerospace Sciences Meeting and Exhibit, Reno, NV, January 6-9, 1992.

[7] J. L. Palmer and R. K. Hanson, "Shock tunnel flow visualization using planar laser-induced fluorescence imaging of $\mathrm{NO}$ and $\mathrm{OH}$," Shock Waves, vol. 4, pp. 313-323, 1995.

[8] J. D. Anderson, Jr., Modern Compressible Flow: With Historical Perspective, $3^{\text {rd }}$ ed. Boston: McGraw-Hill, 2003. 
[9] F.M. White. Viscous Fluid Flow, $2^{\text {nd }}$ ed. New York: McGraw-Hill, Inc., 1991.

[10] J. A. Wilkes, D. W. Alderfer, S. B. Jones, and P. M. Danehy, "Portable Fluorescence imaging system for hypersonic flow facilities," JANNAF Interagency Propulsion Committee Meeting, Colorado Springs, Colorado, December 2003.

[11] K. Bülent Yüceil and M. Volkan Ötügen. "Scaling parameters for underexpanded supersonic jets," Physics of Fluids, vol. 14, no. 12, pp. 4206-4215, December 2002. 